\title{
VALORACIONES DE CALIDAD EN ALIMENTOS ORGÁNICOS Y DE ORIGEN LOCAL ENTRE CONSUMidores DE LA RED COMIDA SANA y CerCANA EN CHIAPAS
}

\author{
Opinions About the Quality of Organic Food Products, and the Origin of the Local Consumer \\ Network "Comida Sana y Cercana" in Chiapas \\ Cynthia Gutiérrez-Pérez \\ Helda Morales \\ Fernando Limón-Aguirre
}

Resumen: El trabajo expone las valoraciones que los consumidores otorgan a la calidad de los alimentos orgánicos de origen local, dependiendo desuposible afinidada la noción de calidad planteada desdeel marconormativo orgánico, ode sisuperponenotrotipodevalores ala horadeelegir. La información fue obtenida mediante un cuestionario aplicado a 50 consumidores asiduos a la red Comida Sana y Cercana en San Cristóbal de Las Casas, Chiapas, y mediante la realización de dos talleres. Los resultados indican que la calidad se define desde aspectos organolépticos —dimensión simbólica asociada al origen local y a la valoración por métodos alternativos de producción - que los consumidores están interesados en el modelo de certificación participativa; y que depositan su confianza principalmente en la relación de cercanía entre productores $y$ consumidores.

Palabras clave: inocuidad, certificación participativa, agroecología.

Abstract: The objective of this work is to expose the opinions that consumers give about the quality of organic food and local food, so that we can know if this opinions are in the same line as the quality notions of the normative organic food or if independent values would take part in the act of choosing food. A questionnaire was made to 50 of the regular consumers of network Comida Sana y Cercana in San Cristóbal de Las Casas, Chiapas, also the information was obtained from two workshops. The results indicate the fact that defined quality. The fact was nutritional, organoleptic, environment and social justice aspects. Also, those consumers show interest for the participative certification, which makes of to consider that confidence in this food is associated mostly with the proximity between producer and consumer.

Keywords: harmless, participative certification, agroecology.

Cynthia Gutiérrez, doctora en Ciencias en Ecología y Desarrollo Sustentable. Egresada de El Colegio de la Frontera Sur, Chiapas. Correo electrónico: cgutierrez@ecosur.mx.

Helda Morales, investigadora del Área Sistemas de Producción Alternativos. El Colegio de la Frontera Sur. Chiapas. Temas de especialización: Manejo y prevención de plagas, agroecosistemas y conservación de sistemas agrícolas. Correo electrónico: helda.bruce@gmail.com.
Fernando Limón, investigador del Área Sociedad, Cultura y Salud, El Colegio de la Frontera Sur, Chiapas. Temas de especialización: Pueblos indígenas, particularmente sus conocimientos culturales, sistemas educativos y médicos, religión e identidades. Correo electrónico: flimon@ecosur.mx.

Enviado a dictamen: 22 de junio de 2012 Aprobación: 05 de noviembre de 2012 Revisiones: 1 


\section{Introducción}

El interés por abordar la relación entre calidad y alimentos tiene que ver con las nuevas exigencias de los consumidores, la mundialización de los mercados agrícolas, la percepción de que la agricultura orgánica mejora los márgenes de los agricultores y de las empresas, y los problemas de salud vinculados al uso de plaguicidas entre los agricultores (Pons y Sivardiere, 2002).

Para la FAO (2000), la calidad puede considerarse una característica compleja de los alimentos que determina su valor o aceptabilidad para los consumidores, señalando que, desde la perspectiva normativa, la calidad está relacionada con los requisitos básicos que deben cumplirse en virtud de las leyes y reglamentos vigentes para que los alimentos sean inocuos y no estén contaminados o adulterados ni se presenten en forma fraudulenta. ${ }^{1}$

Una perspectiva más amplia sobre la calidad de los productos agroalimentarios, de acuerdo con Pons y Sivardiere (2002), tiene los siguientes componentes: características de los alimentos higiénicas, nutricionales, organolépticas-, calidad de uso o de servicio - facilidad de empleo y actitud para la conservación - y calidad psicosocial o subjetiva satisfacción, placer y componentes simbólicos-. En el mismo tenor, Muchnik (2004) señala que la calidad está cada vez más relacionada con otros valores - no necesariamente ligados a la inocuidad- que los consumidores toman en cuenta a la hora de elegir sus alimentos. $^{2}$

En ese sentido, estudios que abordan el comportamiento de los consumidores en relación con los alimentos (Setbon et al., 2005; Schroeder et al., 2007; Tonsor, Schroeder y Pennings, 2008) señalan que hay una tendencia gradual hacia la adquisición de alimentos sanos e inocuos. Asimismo, otros trabajos (Weatherell, Tregear y Allison, 2003; Devcich, Pedersen y Petrie, 2007) refieren que los consumidores se interesan por alimentos orgánicos en función de valores adicionales tales como los beneficios al medioambiente y la justicia social.

Los alimentos orgánicos son resultado de un método de producción considerado inocuo para el medio ambiente ya que se basa en una o varias normas que tienen por objeto conseguir agroecosistemas social y ecológicamente sostenibles (FAO, 2000). Dichas normas abarcan el seguimiento de la totalidad del proceso - cubriendo la producción, la manipulación, la elaboración y la comercialización- por parte de órganos o autoridades debidamente constituidos conocidos como certificadoras.

Por esas características, en los alimentos orgánicos la calidad y la inocuidad están sumamente relacionadas con el proceso mismo de producción por el apego que guardan a los instrumentos de control y los procesos de certificación. Sin embargo, garantizar que la calidad e inocuidad se cumplan ha sido una tarea que ha recaído principalmente en los gobiernos y en instancias internacionales.

Para el caso que nos ocupa hemos de señalar que, además de la posición normativa que regula la calidad y la inocuidad, en nuestro país se ha puesto en práctica una propuesta distinta para garantizar la calidad en el movimiento orgánico. La llamada certificación participativa surge como una alternativa para los pequeños productores que quieren acceder a mercados de comercialización local y que están organizados a través de la Red Mexicana de Tianguis y Mercados Orgánicos. En dicha red, los diversos agentes involucrados - productores, consumidores, promotores y expertos en el tema de la agricultura orgánica - se han propuesto como reto el poner en práctica la certificación participativa. Tal es el caso de Comida Sana y Cercana que pertenece a la Red Mexicana y sobre la cual enfocamos este trabajo.

Comida Sana y Cercana inició con el nombre Canasta Orgánica en 2005, a partir de un pequeño grupo de mujeres que compartían la preocupación por la calidad 
y la seguridad de los alimentos. En septiembre de 2006, el grupo organizó el primer encuentro de productores y consumidores en forma de mercado. A partir de esa fecha funciona a través de un tianguis en San Cristóbal de Las Casas tres veces por semana, constituyendo una experiencia local de comercialización de productos orgánicos, pero sobre todo de productos locales principalmente hortalizas y procesados artesanales-.

Actualmente está constituido por 11 productores agrícolas, seis procesadores artesanales que utilizan insumos limpios, siete procesadores artesanales con insumos convencionales, dos tiendas que comparten la misma filosofía, un productor de varios tipos de carne y un productor de pescados y mariscos.

Este espacio se ha configurado como un lugar donde los consumidores pueden encontrar alimentos que atienden preocupaciones específicas en relación a la producción. Un asunto relevante para el tema que nos ocupa es que en 2010, el tianguis puso en marcha el proceso de certificación participativa para garantizar la calidad de los alimentos que se producen con métodos agroecológicos, artesanales y limpios, y que son elaborados por productores locales.

Uno de los objetivos principales que se encuentra en el origen de Comida Sana y Cercana es su interés por ofrecer alimentos regados con agua limpia. Lo anterior tiene que ver con el hecho de que los alimentos frescos se producen principalmente con métodos convencionales y en algunos casos se riegan con aguas residuales, lo que constituye un problema en términos ambientales y un riesgo para la salud de los productores y consumidores que habitan en San Cristóbal de Las Casas. Al respecto, Kauffer (2006) señala que las aguas residuales de ríos y arroyos que pertenecen a la microcuenca del río Fogótico están formadas por una mezcla de aguas procedentes del drenaje, desechos industriales y agua de lluvia que obviamente no poseen las cualidades exigidas para la actividad hortícola. En ese contexto, la iniciativa del tianguis Comida Sana y Cercana tiene como misión la promoción y el consumo de alimentos que han sido producidos de forma limpia y en beneficio del productor local.

Con la intención de aportar a la temática de la calidad y de las experiencias de producción y comercialización local de alimentos, nos hemos propuesto analizar cómo los consumidores que asisten a dicho espacio de venta expresan sus valoraciones sobre la calidad, con qué la relacionan y qué opiniones tienen sobre los procesos de certificación. La finalidad consiste en reconocer si las opiniones de los consumidores son afines o no a las valoraciones de calidad tradicionalmente planteadas desde el marco normativo orgánico, o si por el contrario anteponen otro tipo de valores a la hora de elegir sus alimentos.

\section{Normas de calidad para los alimentos orgánicos}

A nivel internacional, las normas más difundidas y aplicadas en términos de calidad e inocuidad son las emitidas por la Comisión del Codex Alimentarius, organismo creado en 1963 por la FAO y la OMS para desarrollar normas alimentarias, reglamentos y otros textos relacionados, como códigos de prácticas. Estas normas regulan la producción, elaboración, etiquetado y comercialización tanto de los alimentos producidos bajo el método convencional, como de los producidos bajo el método orgánico.

Los puntos principales que abordan estas normas son: proteger la salud de los consumidores, asegurar prácticas de comercio claras y promocionar la coordinación de todas las normas alimentarias acordadas por las organizaciones gubernamentales y no gubernamentales. En cuanto a su aplicación, cada región y país regula esta materia a través de normativas que toman en cuenta el Codex Alimentarius.

También en el marco internacional se encuentra la Organización Internacional para la Normalización (ISO) creada en 1947 en Ginebra y dependiente de la ONU, con la cual y a través del ISO 9000 se garantizan diversos sistemas de gestión de calidad que han sido adaptados 
al tema de la alimentación (Abdullah, 2007). Las normas ISO se aplican en diversos países y tienen como finalidad facilitar el intercambio comercial. Varias de ellas están relacionadas con la industria alimentaria, como la norma ISO22000, la cual, a través de los Sistemas de Gestión de Seguridad Alimentaria, establece los requisitos que deben reunir todas las partes implicadas en el proceso de la cadena alimentaria.

En México existen principalmente dos agencias que se encargan de la inocuidad de los alimentos frescos y procesados. Dichas agencias dependen de dos secretarías de Estado: la Secretaría de Salud y la Secretaría de Agricultura, Ganadería, Desarrollo Rural, Pesca y Alimentación (SAGARPA). La SAGARPA, a su vez, se encarga de los aspectos de inocuidad a través del Servicio Nacional de Sanidad, Inocuidad y Calidad Agroalimentaria (SENASICA).

En términos generales, la regulación de la inocuidad de los alimentos ha tenido como resultado la implementación de la certificación a fin de garantizar que los sistemas o productos se ajusten a normas técnicas y respondan a necesidades concretas de los consumidores o clientes.

En ese sentido, Pons y Sivardiere (2002) señalan que las ventajas de la certificación en alimentos son: identificar y diferenciar, dar credibilidad mediante la garantía de un organismo de certificación independiente, crear valor agregado a todos los niveles de una cadena de producción determinada, ser mejor conocido y reconocido, y ganar o conservar la confianza de los consumidores. $^{3}$

Una certificación relacionada con la calidad en los alimentos y que ha tomado relevancia en los últimos años es el Análisis de Peligro y Puntos de Control Crítico -o Hazard Analysis Critical Control Points (HACCP) - el cual pone énfasis en los protocolos de higiene durante la elaboración de los alimentos con la finalidad de controlar posibles contaminaciones. Si bien este enfoque no es nuevo, actualmente ha tomado relevancia para la industria alimentaria. ${ }^{4}$ En ese sentido, cabe resaltar que es la Unión Europea la que cuenta con las regulaciones más estrictas a través de diversas normas sanitarias.

En cuanto a los alimentos orgánicos, la certificación surgió, por una parte, como un esfuerzo para proteger los productos del fraude y para garantizar su autenticidad, pero también fue resultado de la exigencia por parte de los consumidores de conocer el proceso de producción de los alimentos llamados orgánicos (Gómez, Gómez y Schwentesius, 1999). Actualmente la certificación se erige como un instrumento de mercadeo en cuyo proceso el productor obtiene un sello que garantiza que un producto, sistema o servicio se ajuste a las normas y estándares de producción, procesamiento y comercialización establecidos por una entidad certificadora. Si bien existen diferentes formas de evaluar la conformidad, el sistema predominante es la certificación por entidades independientes o de tercera parte, es decir, es realizada por una organización independiente de la unidad a certificar (IFOAM, 2009).

Ahora bien, como hemos señalado en la introducción, la calidad en los alimentos orgánicos está muy relacionada con el método productivo de tal manera que, para garantizarla, se regulan diversos aspectos, como el control de peligros químicos a través de la prohibición de plaguicidas, fungicidas, medicamentos veterinarios - antibióticos u hormonas del crecimiento-, aditivos sintéticos e irradiación (IFOAM, 2009).

También se controlan contaminantes ambientales tales como metales pesados y compuestos tóxicos y microbianos, y se regulan los peligros microbiológicos, recomendándose el uso de fertilizantes naturales como el estiércol o los biosólidos y el control de micotoxinas a través de buenas prácticas de alimentación animal (IFOAM, 2009).

En nuestro país la agricultura orgánica se encuentra regulada por la Ley de Productos Orgánicos publicada en el Diario de la Federación en 2006 y por el reglamento de dicha Ley publicado en 2010. Nos parece importante resaltar que el artículo 24 del capítulo cuarto de dicha 
Ley, relativo al sistema de control y certificación, menciona el proceso de certificación participativa como una opción para los pequeños productores. ${ }^{5}$ Esto es relevante para las experiencias locales de producción y comercialización, como en el caso del presente tianguis

Al respecto, en el reglamento se señala que la certificación participativa procede únicamente para la producción familiar o para los pequeños productores organizados, siempre y cuando vendan los productos directamente al consumidor o usuario final. ${ }^{6}$ De igual forma, se recomienda fomentar el consumo de productos orgánicos para promover actitudes de consumo socialmente responsables. ${ }^{7}$

Con la intención de contextualizar y comprender cómo en el tianguis se garantiza la calidad de los productos que se comercializan, en el siguiente apartado exponemos las normas y procedimientos derivados de la certificación participativa llevada a cabo en este espacio.

\section{Certificación agroecológica para alimentos locales}

Dado que la mayoría de los productos que se venden en el tianguis son de origen local y no están certificados como orgánicos por los organismos convencionales, es decir, las certificadoras, el objetivo principal de las normas y procedimientos propuestos por Comida Sana y Cercana es garantizar que todos los productos agropecuarios que se ofrecen en el tianguis cumplan con los criterios de limpieza, sanidad y cercanía. En ese sentido, "son normas que si bien coinciden en algunos aspectos con la certificación orgánica la mayoría están basadas en las condiciones particulares de la región así como en las necesidades, posibilidades y visión de las personas productoras y consumidoras de la Red" (Morales et al., 2011: 1).

Los principios y metas de la certificación participativa son los siguientes:
[...] garantizar calidad y sustentabilidad social, ambiental y económica de los productos que se venden en el tianguis; contribuir a la construcción de una agricultura ecológica, democrática, inclusiva y accesible para todas las personas productoras y consumidoras y crear estructuras por medio de las cuales los actores al interior de la red se puedan apoyar en su lucha para ganar una vida digna en armonía con la naturaleza (Morales et al., 2011: 2).

En esta experiencia se ha puesto énfasis en seguir un modelo de producción agroecológico y no orgánico. Esto es así porque los productores y promotores consideran que el modelo agroecológico responde mejor a las necesidades locales, de manera que:

[...] la certificación agroecológica enfatiza la diversificación de la parcela para mantener suelos fuertes y cultivos resistentes que con el tiempo permitan no utilizar insumos como biofertilizantes. Adicionalmente la certificación agroecológica pone énfasis en la equidad y justicia social y se basa principalmente en el intercambio de saberes de las personas participantes (Morales et al., 2011: 2).

Para hacer viable la propuesta se conformó el Comité de Certificación Participativa, el cual se encarga de asegurar la calidad de los productos a través del cumplimiento de las normas y del seguimiento técnico. Dicho Comité ofrece dos tipos de certificación: la certificación agroecológica participativa en transición y la certificación agroecológica participativa. En ambos casos los productores deben cumplir con los siguientes criterios: comprobar el origen de los productos, producir de manera limpia - esto es, sin agroquímicos y no regados con aguas negras- y cumplir con los requerimientos de la producción agroecológica.

Hasta aquí lo que se ha expuesto corresponde al ámbito normativo relacionado con la regulación de la calidad, tanto en alimentos orgánicos como 
en alimentos de origen local, de acuerdo con lo implementado en Comida Sana y Cercana a través de la certificación participativa. Al respecto, si bien ha dominado el enfoque normativo sobre inocuidad para abordar el tema de la calidad en la producción de alimentos, es posible distinguir que, en el caso de la certificación participativa, se está permitiendo abordar el tema de la calidad desde un enfoque de proximidad de los consumidores con los lugares de producción y con los productores. Por ello, en el próximo apartado nos interesa exponer el tipo de enfoques que abordan el tema de la calidad tomando en cuenta las expectativas de los consumidores al momento de elegir sus alimentos.

\section{Valoraciones de calidad desde el consumidor}

Garantizar alimentos inocuos o seguros para la población a través de acuerdos consensuados que se expresan mediante normativas, es una perspectiva que Muchnik (2004) ha denominado la parte objetiva del concepto de calidad. Sin embargo, el mismo autor explica que para abordar este tema no es suficiente quedarse con el marco normativo. Por ello, propone tomar en cuenta el ámbito individual, es decir, las expectativas de los consumidores a la hora de elegir alimentos.

Esta parte del concepto de calidad más relacionada con las valoraciones de los consumidores es llamada por Muchnik la parte subjetiva. En ella intervienen factores nutrimentales, organolépticos y sensoriales que en conjunto son fundamentales para la elección de los alimentos. ${ }^{8}$

Aunado a los enfoques anteriores nos parece relevante exponer una perspectiva que relaciona el concepto de calidad con el de origen. Años atrás, Espeitx (1996) señaló que el vínculo con el lugar de producción, e incluso con los procedimientos ancestrales, era un aspecto importante a la hora de elegir alimentos. Es decir, que la elección de alimentos se basa en identidades culturales o, también, en el denominado patrimonio alimentario. ${ }^{9}$ Años después esta propuesta fue retomada por Muchnik (2004), quien agregó que la esfera simbólica de los alimentos adquiere un papel relevante para entender las preferencias de los consumidores.

La calidad en relación con los lugares donde se producen los alimentos ha marcado pauta para diferenciarlos según regiones, promoviendo con ello la confianza en los alimentos y un lazo con lo local a través de la relación productor-consumidor. ${ }^{10}$

\section{Metodología}

El estudio se realizó con dispositivos cualitativos y cuantitativos en dos periodos de trabajo de campo. En la primera fase se aplicó en las instalaciones de la red un cuestionario semiestructurado con tres secciones que incluía preguntas abiertas y cerradas, el cual fue respondido por 50 de los consumidores que asisten de forma habitual. ${ }^{11}$ La primera parte del cuestionario contenía preguntas que permitieron caracterizar el perfil sociodemográfico de los consumidores, la frecuencia de compra y las percepciones en torno al precio, la calidad, la confianza y la certificación. ${ }^{12}$ En las preguntas sobre calidad y confianza se utilizó la escala Likert y preguntas abiertas. La segunda fase de trabajo de campo se llevó a cabo en el marco de la certificación participativa. Se facilitaron dos talleres en las instalaciones del tianguis con la presencia de diez de los consumidores que previamente habían respondido al cuestionario. ${ }^{13}$ Ambos talleres fueron convocados con el objetivo de detectar las preocupaciones de los consumidores en torno a la producción agropecuaria y los alimentos procesados, con la finalidad de generar propuestas útiles para mejorar la calidad de los productos ofrecidos. Fueron tres las temáticas generadoras: 1) producción de alimentos y aspectos sociales; 2) producción de alimentos, medio ambiente y salud; y 3) características que los consumidores buscan 
en los alimentos que ofrece el tianguis. Fue el último tema el que permitió profundizar en torno a la calidad y la confianza en tres aspectos: el etiquetado, el envasado y la seguridad e higiene. Sobre la obtención de estos datos es necesario hacer notar que en dichos talleres fue fundamental que dos integrantes de la red Comida Sana y Cercana, quienes fungen como promotores, explicaran a los asistentes su intención de promover sistemas agroecológicos entre los productores, así como la necesidad de implementar la certificación participativa. Se planteaba esta última como una opción para los alimentos de origen local ya que, dadas sus características, no pueden ser certificados como orgánicos.

\section{Resultados de la investigación}

\section{Perfil de los consumidores}

Los consumidores que respondieron el cuestionario tienen un promedio de 40 años de edad. De ellos, 31 son mujeres y 19 hombres. Tienen un promedio de ingresos mensuales de 198 salarios mínimos. ${ }^{14}$ Predomina entre los consumidores el nivel educativo superior (60\%), de los cuales 12 cuentan con licenciatura (24\%) y 18 con posgrado (36\%). Del resto, 16 cuentan con escolaridad media (32\%) y 4 tienen educación básica (8\%).

Todos los informantes viven en la ciudad de San Cristóbal de Las Casas. 38 son mexicanos (76\%) y 12 son extranjeros (24\%). Respecto al tiempo que llevan acudiendo al tianguis, 25 consumidores respondieron que menos de un año, mientras que los otros 25 afirmaron que asisten desde que el tianguis inició (2005). La mitad de los informantes compra el 40\% de sus alimentos en el tianguis, 15 consumidores señalan que compran el 40\% del total de sus alimentos en este lugar y los otros 10 compran sólo el 10\%. Los productos que más adquieren son hortalizas, seguidas de alimentos procesados, productos lácteos y, por último, artículos de aseo personal.
En los siguientes apartados describimos los elementos que se relacionan con la calidad y posteriormente se estudian las respuestas a las tres temáticas propuestas en los talleres. El conjunto de resultados nos permitió visualizar los aspectos que intervienen en la valoración de calidad para el tipo de alimentos que ofrece Comida Sana y Cercana.

\section{Valoraciones sobre calidad, confianza y certificación}

Ante la escala ofrecida en el cuestionario para evaluar la calidad, 44 consumidores (88\%) respondieron que la calidad es buena argumentando que los alimentos cumplen con sus expectativas, mientras que seis informantes (12\%) explican que la calidad es regular y expresan que tienen algunas dudas sobre la forma en la que los alimentos son producidos. En cuanto al tema de la confianza, es decir, si creen o no que los alimentos se producen bajo métodos orgánicos, agroecológicos o limpios, 41 consumidores (82\%) respondieron que confían plenamente en los alimentos que se venden en el tianguis, mientras que $9(18 \%)$ mencionan tener dudas. A partir de estos datos podemos ver que hay dos posiciones diferentes respecto al tema de la confianza y que, en cuanto al tema de calidad, ésta se construye desde tres nociones en ambos grupos.

En primer lugar, se mencionan de una forma reiterada las características organolépticas de los alimentos, refiriéndose a que éstos poseen un mejor sabor, color y aspecto. La segunda noción corresponde a una dimensión simbólica asociada con la producción local. En cuanto a la tercera, las personas encuestadas mencionan la coherencia en el conjunto del sistema productivo. Sobre estos puntos citamos opiniones al respecto:

Hay verduras que se venden en el tianguis que no encuentras en otros lugares, esta variedad es algo que me gusta además de que percibo que tienen un sabor diferente, una zanahoria sabe a zanahoria, las 
que venden en un supermercado no saben a nada (consumidora de 41 años).

Cuando compro platico con los productores y ellos me han dicho que todo es producido en parcelas cercanas, yo creo que eso está muy bien porque pienso que ya no sabemos de dónde traen los alimentos que consumimos normalmente. Acá tengo la seguridad de que son alimentos locales y eso me gusta (consumidora de 29 años).

Los alimentos tienen buena calidad, me han dicho que no tienen agroquímicos y que además están utilizando agua limpia para regarlos, yo veo eso muy bien porque es una propuesta diferente de producción y a su vez nos da a las consumidoras la posibilidad de comprar alimentos que en otros lugares tipo mercados públicos o supermercados, al menos en San Cristóbal, no se consiguen (consumidora de 35 años).

Ahora bien, hemos encontrado de forma generalizada que quienes confían en los alimentos que se venden en el tianguis valoran el hecho de tener un contacto directo con los productores y procesadores. Sin embargo, el grupo que evaluó la calidad como regular señaló que falta información sobre los procesos de producción y de elaboración de los alimentos que se ofrecen en este espacio de venta.

Para los informantes que tienen dudas, el contacto con el productor y procesador no es suficiente, por lo que proponen implementar al interior del tianguis medidas tales como dar información a los consumidores en cada puesto y que el tianguis tenga su propia etiqueta:

Me gustaría saber más sobre la forma en que se produce o se elabora lo que acá se vende, pero si me invitan a una parcela posiblemente no vaya por falta de tiempo. Creo que sería muy bueno que nos dieran más información en los puestos, a lo mejor con fotos o quizá alguna etiqueta que sea sólo del tianguis, con ello tendría confianza en lo que compro (consumidora de 36 años).
Dado que uno de los objetivos de la investigación es conocer si los consumidores relacionan calidad con certificación, les preguntamos si conocen la certificación como una medida para garantizar confianza en los alimentos y, en caso afirmativo, quiénes consideran que son los actores que deberían participar en ese proceso. Los resultados indican que 44 consumidores (88\%) reconocen de forma exacta en qué consiste la certificación en alimentos orgánicos, mientras que seis (12\%) mencionaron no conocer este mecanismo.

Respecto a la pregunta sobre quiénes son los actores más indicados para participar en los procesos de certificación, los resultados fueron los siguientes: 30 informantes (60\%) respondieron que en los procesos de certificación deben participar tanto productores como consumidores. 12 consumidores (24\%) consideran que los centros académicos son los más indicados para echar a andar esos procesos; cinco consumidores (10\%) señalaron que las certificadoras son suficientes; y sólo tres (6\%) afirmaron que el gobierno es el actor indicado para garantizar el proceso.

Estos datos resultan relevantes para el proceso mismo de la certificación participativa, ya que los consumidores tienden a considerar que el productor, el consumidor y los centros de investigación pueden desempeñar un importante papel para avalar la calidad de los alimentos, mientras que dan menor credibilidad a la intervención del gobierno e incluso a las certificadoras de alimentos orgánicos.

Los datos de este apartado revelan dos aspectos importantes para abordar el tema de la calidad. El primero es que los resultados indican una dimensión subjetiva en los informantes, en la que destaca la dimensión organoléptica y simbólica, lo cual coincide con el marco conceptual propuesto por Muchnik (2004). El segundo aspecto es que la dimensión objetiva de la calidad basada en las normas y en la regulación del gobierno - en este caso el papel de las certificadoras para los alimentos orgánicos-, si bien se menciona, no constituye un discurso generalizado. 
Consideramos que este tipo de discurso entre los consumidores tiene que ver con tres situaciones. La primera es que estos consumidores se encuentran sensibilizados en torno al tema de la producción local de alimentos, esto es, que previamente han participado en actividades organizadas por el propio tianguis a través de sus promotores y productores. La segunda es que, dado el perfil que tienen de alta escolaridad y por su mayor información sobre lo orgánico, este tipo de consumidores ha desarrollado una posición política respecto a la revalorización de la agricultura, los productores y el medioambiente. Por último, la aceptación y credibilidad hacia este tipo de alimentos se relaciona con el hecho de que el nivel de confianza se basa en las relaciones productor-consumidor, lo que en sí representa una forma diferente de consumo.

En el siguiente apartado describimos de forma más detallada la posición de los consumidores obtenida en los talleres sobre los elementos arriba señalados.

\section{Aspectos de interés para garantizar la calidad en los alimentos de origen local}

Con base en la información obtenida en los talleres hemos resumido en la tabla 1 los aspectos que resultaron de mayor interés para los consumidores en torno a los alimentos agropecuarios y procesados, y su relación con los aspectos sociales, ambientales y de salud. Hemos agregado el nivel de prioridad que dieron a cada aspecto, y en una columna final se destacan los puntos relacionados con la higiene y la seguridad.

La información de la tabla 1 muestra, por un lado, preocupaciones en torno al manejo de alimentos y a la forma en que éstos son producidos o preparados $y$, por otro lado, revela temas que a los consumidores les gustaría que fueran tomados en cuenta en la certificación participativa. Los resultados muestran nuevamente la revalorización del trabajo agrícola - lo cual coincide con lo encontrado en los cuestionarios-. Se propone reconocer los alimentos que se venden en el tianguis con un pago mejor y más justo porque son producidos bajo métodos alternativos. Esta revalorización de la agricultura se relacionó también con la propuesta de que el equipo promotor genere capacitaciones para que los productores cuenten con cálculos de producción y diversifiquen parcelas para el autoconsumo. Asimismo, se sugirió que los consumidores habituales den una cuota voluntaria anual o semestral para ayudar a disminuir los costos de transporte, además de promover el intercambio de alimentos entre los mismos productores. Las celebraciones y festejos se mencionaron como medidas que permitirían fortalecer las relaciones entre productores y consumidores.

Las respuestas a las preguntas sobre la alimentación y su relación con el medioambiente y la salud muestran también varios aspectos que deben tomarse en cuenta para que los alimentos sean percibidos como sanos y seguros por los consumidores. Una de las respuestas más recurrentes se refiere al manejo de desechos. Este punto se considera fundamental porque los desechos pueden contaminar y afectar la calidad de los productos, así como ocasionar problemas de salud a los productores y consumidores. En relación con este tema proponen establecer una casita de reciclado en las instalaciones del tianguis, que los productores reciban capacitaciones sobre elaboración de compostas y que se ofrezcan opciones de reciclaje.

Otra problemática que preocupa a los entrevistados es cómo garantizar la calidad del agua que se utiliza para el riego. Al respecto, se identificaron tres acciones para implementar en campo: verificar fuentes de agua, realizar estudios de presencia de coliformes y de elementos químicos, y verificar que en las parcelas no se utilicen agroquímicos.

Otro elemento importante que interviene en la construcción del concepto de calidad tiene que ver con la búsqueda de información sobre cuestiones de salud que les preocupan. En este sentido, los informantes señalan que es importante contar con información clara 
y suficiente sobre los ingredientes que se utilizan en los alimentos procesados. Argumentan que esa información les permitiría evaluar su consumo para casos de alergias, intolerancias o prohibiciones médicas.

Por otro lado, la identificación de materias primas les permitiría conocer si se trata de alimentos de origen orgánico o de producción limpia, y si tienen o no conservadores. Este aspecto está muy vinculado con el uso de materiales reciclados para envasado y con la creación de una marca para los alimentos del tianguis - propuesta que también encontramos en los cuestionarios-. Sobre este asunto resalta también el interés de que los alimentos procesados sean conservados mediante técnicas alternativas consideradas menos dañinas.

Por último, en relación con la higiene y la seguridad, los resultados responden a preocupaciones sobre el manejo de ciertos alimentos como la carne, un producto de reciente ingreso al tianguis, pero también sobre el manejo de alimentos sin empaque. Sobre este asunto, las respuestas están muy ligadas a aspectos de inocuidad tales como el uso de cofias y guantes, y a garantizar que quien produce o cocina se encuentre en buenas condiciones de salud.

\section{Conclusiones}

Las normas de calidad en términos de inocuidad han sido abordadas de forma tradicional en el ámbito de la gran industria alimentaria, pero se ha dejado de lado en la producción a menor escala y en la producción bajo métodos alternativos. Si bien los alimentos orgánicos han abierto camino para reflexiones sobre las formas de garantizar la calidad de esos alimentos, hemos visto cómo, en el caso de la red Comida Sana y Cercana, la idea de calidad se construye a partir de necesidades específicas de los consumidores, lo cual los hace más propensos a identificarse con el modelo de la certificación participativa. Es decir, que si bien los consumidores saben de qué se trata la certificación de productos orgánicos, se muestran más identificados con el proceso de certificación participativa al señalar que en la certificación deberían participar productores, consumidores e incluso centros académicos.

En ese sentido, el proceso de la certificación participativa es un mecanismo que está permitiendo fortalecer de forma gradual las relaciones de confianza entre los involucrados. Se trata de un proceso de certificación muy influido por la representación que los productores y los consumidores tienen de los alimentos de calidad. Por ello, tener en cuenta aspectos subjetivos resulta un enfoque necesario y adecuado al contexto en el que se desenvuelven este tipo de espacios de producción y comercialización locales.

En el caso analizado, la noción de calidad es construida desde tres nociones: valoraciones de tipo organoléptico, dimensión simbólica asociada a la producción local y confianza en el uso de métodos de producción alternativos a los convencionales. De estos aspectos, dos tienen que ver con una dimensión subjetiva, mientras que el último estaría más apegado a los requisitos planteados en las normas de calidad para alimentos orgánicos. Ahora bien, los talleres muestran que el tema de la inocuidad es importante también para generar confianza en los alimentos que se ofrecen. La información indica que existen preocupaciones relacionadas con este tema, sobre todo en el caso de los alimentos procesados y de los de nuevo ingreso, como es el caso de la carne.

Los puntos de vista emitidos por los consumidores y que eventualmente están más relacionados con la inocuidad nos indican, por un lado, que si bien hay un nivel de confianza muy elevado de los consumidores hacia el tianguis, es necesario tomar algunas previsiones para cubrir necesidades muy específicas en términos del manejo de los alimentos. Sobre este tema, la necesidad de información por parte de los consumidores puede ser vista como una oportunidad para que el tianguis genere materiales de difusión, etiqueta propia y visitas a las parcelas de producción, es decir, se trata de una 
experiencia en proceso de fortalecerse y de generar cambios en las relaciones con los consumidores.

Queremos destacar que, si bien el caso expuesto corresponde a una experiencia local y muy concreta sobre las apreciaciones de un grupo de consumidores, los hallazgos de esta investigación pueden ser relevantes para otros sectores, ya que en un estudio previo (Gutiérrez et al., 2012) se identificaron preocupaciones similares entre los consumidores que asisten a otros espacios de venta, tales como mercados públicos y supermercados. Aunado a este punto, la problemática en cuanto al uso extendido de aguas residuales en la producción de alimentos frescos afecta a la mayoría de la población; por tanto, si el tianguis tuviera una mayor difusión podría poco a poco abarcar a un mayor número de consumidores y tener un impacto social más elevado.

Finalmente, coincidimos con trabajos como los de Goodman y Dupois (2002) y Winter (2003) respecto a que la perspectiva que enfatiza lo local replantea una nueva forma de producción rural y una reconceptualización de la noción de calidad, la cual se orienta hacia una nueva forma de hacer política con impacto en las actitudes futuras de compra de los consumidores.

\section{Notas}

1 22 a Conferencia Regional de la FAO para Europa, Oporto.

${ }^{2}$ Años atrás, Mainguy (1989) ya había abordado estos aspectos al plantear la calidad en función de cuatro "S": satisfacción, seguridad, salud y servicio. Esta propuesta tuvo un fuerte impacto en el desarrollo de parámetros para el tema de calidad en el sector agroalimentario francés.

${ }^{3}$ Cabe mencionar que los organismos de certificación son a su vez acreditados mediante la norma ISO 65, en la cual se plantean los requisitos que deberán tener los interesados para conformarse como organismo certificador de tercera parte (Pons y Sivardiere, 2002).
${ }^{4}$ De acuerdo con Abdullah (2007), este tipo de certificación se originó a finales de la década de los sesenta, cuando la NASA solicitó a la compañía Pillsbury la producción y suministro de alimentos seguros para astronautas.

5 Artículo 24, título cuarto, "Del sistema de control y certificación de productos orgánicos". Capítulo primero de los organismos de certificación y la certificación.

${ }^{6}$ Artículo 14, capítulo IV, "De la certificación orgánica del reglamento de la Ley de Productos Orgánicos".

${ }^{7}$ Artículo 38, apartado 3, título sexto, "De la promoción y fomento". Capítulo único.

8 Duhart, en su trabajo "La calidad en el sistema agroalimentario: lecciones de la experiencia en Francia", hace una clasificación muy similar, pero la nombra de otra manera. Las calidades horizontales son las que corresponden al individuo y las calidades verticales son aquellas que tienen que ver con nociones de calidad consensuadas - técnicas o normas nacionales e internacionales, entre las que se incluye la certificación de orgánicos- Disponible en: http://www.agbioinfo. com/literatura/agricultura/cesaa.pdf [16 de mayo de 2012].

9 Francia fue uno de los primeros países europeos en implementar una política de signos oficiales de identificación de la calidad y del origen de productos alimentarios, entre los cuales se encuentran: apelación de origen controlado, etiqueta roja, agricultura biológica y certificación de conformidad, los cuales en conjunto corresponden a un 19\% del consumo de alimentos a nivel familiar.

${ }^{10}$ Sobre este tema, ver los movimientos Slow Food y Terra Madre que se fundan en el concepto de ecogastronomía, el cual permite reconocer la fuerte conexión entre comida, terruño y planeta. De acuerdo con estos movimientos, la alimentación debe ser buena, limpia y justa. Disponible en http://www.slowfood.com y http:// www.terramadre.org [28 de mayo de 2012].

${ }^{11}$ El promedio de personas que asiste al tianguis es de 100.

${ }^{12}$ La segunda parte del cuestionario abordó el tema de las representaciones sociales en torno a los 
alimentos orgánicos, y la última sección las actitudes y disposiciones para adquirir este tipo de alimentos. La información de ambos apartados fue publicada. Ver referencia completa en bibliografía del artículo de Gutiérrez et al. (2012).

13 Además de los talleres se hicieron tres visitas a dos parcelas y una a procesadores. Los talleres con consumidores y las visitas fueron facilitados por Gabriela Ortiz, José Ruiz y Cynthia Gutiérrez. La información obtenida fue complementada con la del equipo que facilitó los talleres con productores, lo que en conjunto sirvió para la conformación de las normas y procedimientos a los que nos hemos referido.

${ }^{14}$ De acuerdo con la Comisión Nacional de Salarios Mínimos del Diario Oficial de la Federación (2012), para la región C - a la cual pertenece el estado de Chiapasel salario mínimo es de 59.08 pesos por ocho horas de trabajo.

\section{Bibliografía}

Abdullah Jasim, Mahmud (2007), Normas de calidad en la industria alimentaria nivel europeo e internacional. Implantación, problemáticas y desarrollo, (tesis de doctorado), Departamento de Nutrición y Bromatología. Universidad de Granada, España.

Devcich, Daniel A., Irene K. Pedersen y Keith J. Petrie (2007), "You eat what you are: Modern health worries and the acceptance of natural and synthetic additives in functional foods", en Appetite, vol. 48, núm. 3, pp. 333-337.

Espeitx Bernat, Elena (1996), "Los nuevos consumidores o las nuevas relaciones entre campo y ciudad a través de los productos de la tierra", en Agricultura y Sociedad, núm. 80-81, julio-diciembre, pp. 83-116.

FAO (2000), Inocuidad y calidad de los alimentos en relación con la agricultura orgánica, 22ª Conferencia Regional de la FAO para Europa, Oporto, Portugal. Julio.

Goodman, David y E. Melanie DuPois (2002), “Knowing Food and Growing Food: Beyond the Production- consumption debate in the sociology of agriculture", en Sociología Ruralis, vol. 42, núm. l, enero, pp. 5-22.

Gómez Tovar, Laura, Manuel Angél Gómez Cruz y Rita Schwentesius (1999), Desafíos de la agricultura orgánica, comercialización y certificación, México: UACH/ CIESTAM/Mundi Prensa.

Gutiérrez Pérez, Cynthia, Esperanza Tuñón Pablos, Fernando Limón Agurre et al. (2012), "Representaciones sociales de los alimentos orgánicos entre consumidores de Chiapas", en Revista Estudios Sociales, vol. XX, núm. 39, enero-junio, Hermosillo, Sonora: Centro de Investigación en Alimentación y Desarrollo, pp. 100-129. IFOAM (2009), Los principios de la agricultura orgánica, 〈http://www.ifoam.org/about_ifoam/pdfs/POA folder_spanish.pdf > [10 de junio de 2010].

Kauffer Michael, Edith (2006), "La ley de aguas nacionales frente a las prácticas indígenas: ¿una historia de desencuentros?, en Soares, D. et al. (coords.) Gestióny cultura del agua, tomo 1, México: SEMARNAT/IMTA/ Colegio de Posgraduados, pp. 215-236.

Mainguy, Pierre (1989), La qualité dans le domaine agroalimentaire. Rapport de mission, Ministère de l'Agriculture et de la Forêt, Secrétariat d'Etat chargé de la consommation, París.

Morales, H., M. Altuzar, B. Ferguson et al. (2011), Normas y procedimientos de la certificación agroecológica participativa, México: Red de productores y consumidores Comida Sana y Cercana.

Muchnik, José (2004), "Identidad territorial de los alimentos: alimentar el cuerpo humano y el cuerpo social", en Congreso Internacional Arte Agroindustria Rural y Territorio, Toluca, México.

Pons, Jean-Claude y Patrick Sivardiere (2002), "Certificación y acreditación", en Manual de capacitación. Certificación de calidad de los alimentos orientada a sellos de atributos de valor en países de América Latina, Francia y Chile. Francia y Santiago de Chile. ECOCERT/FAO, pp. 11-18. Setbon,M.,J.RaudeyC. Fischler,etal.(2005), "Riskperception of the mad cow disease in France: Determinants and consequences", en Risk Analysis, vol. 25, pp. 813-826. 
Schroeder, Ted, Glynn Tonsor y Joost Pennings, et al. (2007), "Consumer food safety risk perceptions and attitudes: Impacts on beef consumption across countries", en The Journal of Economic Analysis and Policy, vol. 7, artículo 65.

Tonsor, Glynn, Ted Schroeder y Joost Pennings (2008), "Factors Impacting Food Safety Risk Perceptions", en JournalofAgricultural Economics, vol. 60, núm. 3, pp. 625-644.
Weatherell, C., A. Tregear y J. Allison (2003), "In search of the concerned consumer: UK public perceptions of food, farming and buying local", en Journal of Rural Studies, vol. 19, pp. 233-244.

Winter, Michael (2003), "Embeddedness, the new food economy and defensive localism", en Journal of Rural Studies, vol. 19, pp. 23-32. 
Tabla 1

\begin{tabular}{|c|c|c|c|}
\hline Temas & Sector agropecuario & Sector procesados & Higiene y seguridad \\
\hline $\begin{array}{l}\text { Alimentación y aspectos } \\
\text { sociales. }\end{array}$ & 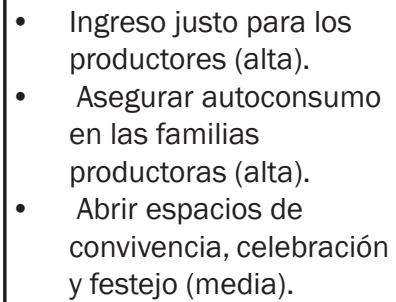 & $\begin{array}{l}\text { Asegurar buen estado } \\
\text { de salud entre quienes } \\
\text { procesan (alta). } \\
\text { Ampliar presencia } \\
\text { del tianguis en otros } \\
\text { sectores (media). }\end{array}$ & $\begin{array}{l}\text { - Mejorar el espacio de } \\
\text { zona de alimentos en el } \\
\text { tianguis (alta). }\end{array}$ \\
\hline $\begin{array}{l}\text { Alimentación y medio } \\
\text { ambiente. }\end{array}$ & $\begin{array}{l}\text { - Manejo de desechos } \\
\text { (alta). } \\
\text { Uso de agua limpia para } \\
\text { riego (alta). }\end{array}$ & $\begin{array}{l}\text { - Manejo de desechos } \\
\text { (media). }\end{array}$ & $\begin{array}{l}\text { - } \quad \text { Prohibir el uso de } \\
\text { bolsas de plástico (alta). } \\
\text { Utilizar materiales } \\
\text { reciclados (alta). } \\
\text { Asignar zona para } \\
\text { separación de desechos } \\
\text { (alta). }\end{array}$ \\
\hline Alimentación y salud. & $\begin{array}{ll}\text { - } & \text { Evitar uso de } \\
\text { agroquímicos (alto). } \\
\text { Manejo adecuado de } \\
\text { carne (medio). }\end{array}$ & 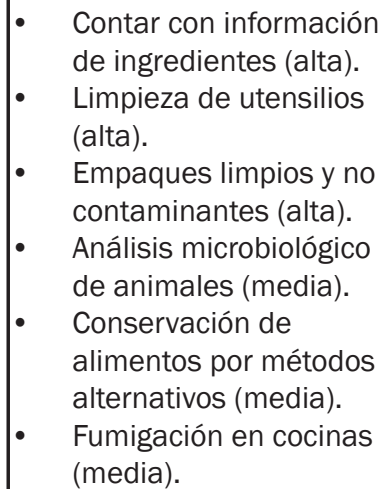 & $\begin{array}{l}\text { - Uso de guantes, cofias } \\
\text { y pinzas para alimentos } \\
\text { no empaquetados } \\
\text { (alta). }\end{array}$ \\
\hline
\end{tabular}

Fuente: Trabajo de campo. Talleres de certificación participativa con consumidores (2011). 